\title{
Integrating Decompositional Event Structures into Storylines
}

\author{
William Croft, Pavlína Pešková, Michael Regan \\ MSC 032130 Linguistics \\ 1 University of New Mexico \\ Albuquerque NM 87131-0001, USA \\ \{wcroft, pavlinap, reganman\}@unm. edu
}

\begin{abstract}
Storyline research links together events in stories and specifies shared participants in those stories. In these analyses, an atomic event is assumed to be a single clause headed by a single verb. However, many analyses of verbal semantics assume a decompositional analysis of events expressed in single clauses. We present a formalization of a decompositional analysis of events in which each participant in a clausal event has their own temporally extended subevent, and the subevents are related through causal and other interactions. This decomposition allows us to represent storylines as an evolving set of interactions between participants over time.
\end{abstract}

\section{Introduction}

Stories are typically represented as a set of events and temporal relations among events (Caselli and Vossen, 2012). However, events are frequently given a decompositional analysis in linguistics, as surveyed in Levin and Rappaport Hovav (2005), and also in computational linguistics, for example Narayanan (1997). Many of the event decompositions in linguistics do not explicitly represent the temporal dimension, and distribute participants across different event components. Such representations do not lend themselves well to integration with storyline analysis.

The event decomposition proposed in Croft (2012) and applied to event annotation in Croft et al. (2016) is more suited to integration with storyline analysis. Croft's analysis of events explicitly represents time as a geometric dimension, as part of the representation of aspect-the structure of events as they unfold in successive phases over time. In addition to explicit representation of the temporal dimension, Croft introduces a second dimension, qualitative states, to model change over the course of the event. These two dimensions allow one to represent directly the pre-state and post-state of events (Im and Pustejovsky, 2010; Segers et al., 2015), as different states on the qualitative dimension, and as different points of time in the temporal dimension.

Croft's analysis also decomposes events into distinct subevents for each participant. The subevents represent directly the interactions of participants, instead of representing them indirectly and incompletely by semantic role labels. This decomposition allows for a smoother integration of complex event structure with story networks, albeit with a reinterpretation of the structure of stories.

The decompositional model of events allows us to consider an alternative model of the structure of stories. In this model, stories are made up of participant histories, that is, the participant's existence through time. A participant history is in turn made up of subevents, namely the states and processes that the participant has or undergoes during each interval of time. The participant histories are related to each other through participant interactions, that is, subevent relations within events, at certain times. This alternative decomposition of stories is also suggested by van Erp et al. (2014), who use a modified metro map visualization (Shahaf et al., 2012), with participants as "lines" and events as "stations". This alternative model can be more fully realized using a decompositional analysis of events in which each participant has its own subevent.

This alternative decomposition of stories is also more independent of the linguistic expression, in which events are realized as simple clauses with one or more argument phrases denoting partici- 
pants, and interclausal syntax expresses temporal and other relations between events. Thus, it represents a story structure that must be derived indirectly from the linguistic expressions. In this paper we present a formalization of this alternative decomposition of stories and events, and show how this formalization can be used to construct a visualization of stories as an evolving network of interactions among participants over time.

\section{Event Decomposition and Annotation}

\subsection{Subevents and Aspect}

In Croft's decompositional analysis, each participant has its own subevent. Each subevent consists of a sequence of temporal phases, representing how the subevents unfold over time. A subevent is made up of phases that are defined on two dimensions, time and a dimension of qualitative states that can be used to define different types of states and processes. That is, instead of representing the qualitative states and changes of an event as an atomic predicate, a predicate is analyzed as a path through a one-dimensional quality space over time. Obviously, a one-dimensional representation of qualitative states/changes is a simplification, but it is an advance on analyses in which the qualitative event structure is left unanalyzed as the verbal "root" (Levin and Rappaport Hovav, 2005).

Subevents have an aspectual type. Semanticists have identified a number of different aspectual types, most of which can be analyzed as special cases of Vendler's categories of states, achievements, activities and accomplishments (Vendler, 1957; Croft et al., 2016). States lack change on the qualitative dimension. Some states are inherent properties of an individual (She is French), while others are reversible (The window is open) or irreversible (The window is broken), and still others exist only in a point of time (The sun is at its zenith).

Achievements represent a transition, construed as instantaneous, from one qualitative state to another. Directed achievements transition to a result state (The window broke), while semelfactives (called cyclic achievements by Croft) transition to the result state and back to the initial state (The light flashed). Accomplishments represent a gradual change on a qualitative dimension over time, attaining a natural endpoint. Incremental accomplishments represent a measurable, monotonic change (She ran into the gym), while nonincremental accomplishments describe an activity that is not monotonic before achieving the result state (He repaired the computer). Activities represent change that does not have a natural endpoint. Directed activities represent a monotonic change (The balloon rose), while the change described by undirected activities is nonmonotonic (The fans were dancing.).

We argue that there is another Vendler-like category: processes that stop, returning to the base state. These events, which we call endeavors, are temporally bounded, but not by reaching a natural endpoint. They may be directed or undirected. Endeavors are not lexicalized as such in English, but certain subevents in complex events are endeavors. In Russian, there are lexicalized endeavors (Forsyth, 1970). Undirected endeavors are derived from undirected activities with the prefix po-, as in On po-spal posle obeda 'He had a sleep after dinner'. Directed endeavors are derived from directed activities with the prefixes pri-, pod-, and nad-, as in On pri-otkryl dver' 'He opened the door a little'.

Participant subevents cause other participant subevents; this is the domain of force dynamics (Talmy, 1988). Croft (2012) extends Talmy's notion of force dynamics to cover a wide range of asymmetric relations between participants. The commonest noncausal interaction is a spatial relationship between two entities, the figure and the ground, following Talmy (1983).

Many different event types are discussed in the linguistic semantic literature: caused motion, application, emission, change of state, and so on. Construction grammarians argue that these schematic event types represent the meanings of argument structure constructions (Goldberg, 1995, 2006).

\subsection{Force Dynamics and Types of Qualitative Change}

Croft et al. (2016) propose an analysis of the semantic types of argument structure constructions in terms of force-dynamic relations between participants, causal and noncausal, and the type of change that the theme participant undergoes. Among the most common types of force-dynamic relations are Force, the prototypical physical transmission of force relation; Constrain, Talmy's "causation of rest/stasis" (Talmy, 1988); and Path, the spatial figure-ground relation.

Croft et al. define four types of physical 
changes, based in part on different types of incremental theme (Dowty, 1991; Hay et al., 1999). The simplest change subevent of the affected entity is a change of state of the entity, that is, a change in a scalar property of the entity as a whole (Hay et al., 1999).

Events involving change in a spatial figureground relation proceed in two different ways. Motion events of various kinds, such as The boy ran across the road, define a spatial path on the qualitative dimension that the figure traverses as a whole; for this reason Dowty (1991) calls the figure a "holistic theme".

Application, removal, combining and separating events, such as The man picked pears from the tree, define a mereological change in the location of figure on the qualitative dimension; this is Dowty's incremental theme proper. Covering and uncovering events, such as I buttered the toast with hazelnut butter and They stripped the trees of bark, differ from application and removal events in that the incremental change is conceptualized as happening to the ground object (toast, trees) rather than the figure.

Croft et al. define another type of theme change, which they call Design for creation of an object with a certain identity, for events of creation (They built a shelter), formation (She carved a toy out of a stick) and replication (He scanned the article).

We identify another type of physical change not described by Croft et al. (2016): internal change of a single participant, such as The flag fluttered. Internal events often also express a locative relation: The flag fluttered (over the fort). Finally, simple static location is included as an internal event type, albeit static: The flag is over the fort.

The relations between subevents and properties of subevents summarized above cover a large range of the inventory of physical processes expressed by simple verbs in English. There are of course many other events involving mental processes and other interactions between humans and other entities (perception, cognition, emotion, intention, attention, etc.), and many other events involving social interactions, which remain to be analyzed in this decompositional framework.

\subsection{Annotation and Visualization of Aspect and Force Dynamics of Events}

We proposed an annotation scheme for annotating clauses with their aspectual type and force- dynamic type of change, based on the verb, tenseaspect construction, and argument structure construction (Croft et al., 2016). We retain this aspect annotation, with the addition of directed and undirected endeavors. The full list of aspectual types is found in Table 4 in the Supplementary Material, which also includes their formalization (see section 3). We are developing a revised annotation of aspectual types, a notoriously difficult area of linguistic semantics, that we believe will be simpler to use by annotators, yet still captures all of the distinctions in Table $4 .{ }^{1}$

The force dynamic annotation scheme in Croft et al. (2016) annotates only the type of change undergone by the theme participant in the clausal event. The revision and extension of the annotation scheme described above is found in Table 5 in the Supplementary Material. The theme change may be externally caused; annotation for external and internal cause can be found in Table 6 in the Supplementary Material.

A complete annotation of the structure of a clausal event consists of three annotations: the aspectual type (ideally, the fine-grained classification in Table 4); and for the force dynamics, the external/internal cause (Table 6) and the theme change type (Table 5).

We applied this annotation scheme, with the modifications described in this section, to clauses in three Pear Stories (Chafe, 1980). The Pear Stories are a set of oral narratives produced by speakers after viewing a short film which was designed to analyze patterns of verbalization. Since there is no language in the film, the narratives mostly encode physical events of the type already analyzed by Croft et al. We chose the Pear Stories since this allowed us to easily annotate most of the events in the narrative. We believe that the oral narratives share significant narrative structure with news stories. However, temporal ordering of events is much more regular in the Pear Stories.

Relations between clausal events in the Pear Stories were annotated using predicates from the temporal interval calculus (Allen, 1984; Mani and Pustejovsky, 2012). Temporal relations expressed by adverbial clauses and coordination are represented adequately for our purposes by the temporal interval calculus predicates. The temporal interval calculus predicates that expressed inter-

\footnotetext{
${ }^{1}$ The revised aspect annotation scheme, and annotation guidelines for aspectual and force dynamic annotation, will be found at http://www. unm. edu/ w wcroft.
} 


\begin{tabular}{|llll|}
\hline Event & FD 1 & FD 2 & Aspect \\
\hline And he comes down, . . from the ladder, & Self-volitional & Motion & Incremental Accomplishment \\
[1.1] and he's wearing an apron, & Volitional & Constrain & Undirected Activity \\
And he dumps them [.45] into some baskets . . & Volitional & Apply & Incremental Accomplishment \\
\hline
\end{tabular}

Table 1: Annotation of a passage from the Pear Stories

clausal relations occurring in the Pear Stories narratives that were annotated are Before (BEF), After (AFT), Meets (MEET), Equal (EQ), Overlap (OVER), and Contains (CONT). Coreference relations between participants across events were also annotated by using the same values for recurrent individuals in the constructional annotation (not shown in Table 1).

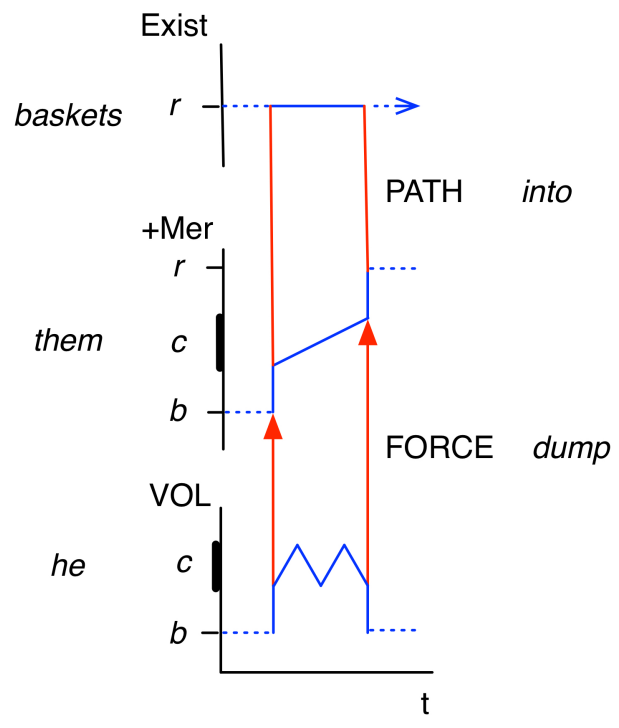

Figure 1: Graphic representation of decompositional event structure for And he dumps them into some baskets

A sample annotation is given in Table 1. From this annotation, we construct predicate calculus representations of the decompositional event structure based on the formalization in section 3. From the formal representation, we automatically generate a graphic representation of a clausal event. Figure 1 shows the graphic representation of the third sentence in Table 1. The graphic representation is a modified version of those found in Croft (2012). In particular, we add structure to the $q$ dimension (the vertical dimension) for each participant, as described in section 3.

Adding the annotation of temporal relations between clausal events allows us to generate a visualization of the fragment of the story in Table 1; see Figure 4 and the discussion in section 4 .

\section{A Formalization of Event Structure}

Here we formalize the idea that stories are made up of participant histories that interact over time. This view of story structure informs the formalization of the individual events in a story that express the participant interactions. Since event structure is complex, almost all of our attention here will be focused on the event structure formalization. The formalization expands the annotation to formulas that allow for inference about events and their participants, and allow for visualizations of the structure of events and the structure of stories.

\subsection{Aspect and the Interval Calculus}

Our formalization uses the interval calculus for both the temporal and qualitative dimensions (Allen, 1984; Mani and Pustejovsky, 2012), and the commonsense knowledge axioms of Gordon and Hobbs (2017). ${ }^{2}$ Since event decomposition involves many composite entities, we use the notational simplification of $x=a+b$ to describe a composite entity $x$ with exactly $a$ and $b$ component entities, that is, CompositeEntity $(x) \&$ Componentof $(a, x) \&$ Component-of $(b, x) \& a \neq b \&((y \neq a$ $\& y \neq b) \supset \neg$ Component-of $(y, x)$ ); likewise for composite entities with more than two component entities. The notation $x=a$ indicates equality, that is, there is exactly one component to the composite entity. However, we will use $\operatorname{Equal}(i, j)$ for interval equality following Allen (1984).

We begin with the formal analysis of subevents and their participants. Each participant is identified with its own subevent. A participant is modeled as a history, namely, the states and changes that a participant has, performs or undergoes over time. The identity of a participant as an individual is expressed by the unity of the participant history.

A subevent is a component of a participant history. The subevent consists of qualitative states and changes of the participant during a time interval of the participant history. We model the quali-

\footnotetext{
${ }^{2} \mathrm{We}$ use the axioms that are presented at http://www.isi.edu/ hobbs/csk.html, which are basically identical to the axioms that will appear in Gordon and Hobbs (2017).
} 
tative structure of a subevent by the qualitative dimension $q$ orthogonal to the time dimension $t$.

Different verbs or predicates define different relevant qualitative states for each participant subevent. Hence each subevent has a distinct set of qualitative states. One can consider each predicate's set of qualitative states as an interval on the $q$ dimension. Alternatively, each predicate can be thought of as representing a distinct qualitative dimension (see section 4). Where necessary, we will distinguish qualitative state dimensions for different predicates, for example for different subevents of a multiparticipant event, as $q_{1}, q_{2} \ldots$

Following Allen (1984), we represent "points" in time as very small intervals. Specifically, we define a "point" interval as an interval that does not contain a smaller interval, that is, $\operatorname{Pnt}(i) \equiv$ $(\neg \exists j)$ During $(j, i)$. Extended (Ext) intervals are not punctual. One reason for treating points as the smallest intervals is that an event that is construed as occurring in an "instant" (The bridge collapsed) may also be construed as occurring over an interval (The bridge is collapsing). We would represent these two construals as both occurring over intervals with different granularities (Hobbs, 1985a) such that for the coarser-grained temporal metric, there are no smaller intervals than the event interval, but for a finer-grained temporal metric, there are. (We have not yet modeled granularity shifts.)

\subsection{The Structure of the Qualitative Dimension}

We analyze the structure of the qualitative dimension $q$ for each subevent also using the interval calculus, which can be generalized beyond time (Mani and Pustejovsky, 2012; Hobbs and Pan, 2004). Verbs and other predicates impose more specific structure on $q$.

We distinguish four types of qualitative dimensions that capture the potential variation of qualitative states defined by predicates over time. Inherent predicates cannot vary over time for a participant; for example one cannot start or stop being French. Hence only one point is defined on $q$, which we label $r$. Complementary predicates can vary between applying or not applying to a participant; for example a window can be either whole or broken. Only two points are defined on $q$, a "base state" $b$ called a "rest state" in Croft (2012), and the "result state", also labeled $r$. Graded pred- icates vary dynamically in their states beyond the base state $b$; for example, one can either dance or not dance, but dancing involves various changes on a dimension of bodily movements. Graded predicates involve the base state $b$ and a continuous interval $c$ for the process. Finally, telic predicates such as entering a room have a base state $b$ (not being in the room), the central interval of dynamically varying states $c$ (the entering movement), and a result state $r$ (being in the room).

The types of predicates are defined in Table 3 in the Supplementary Material. The structure of a telic predicate is illustrated in Figure 2.

\subsection{Phases and Subevents}

A phase is defined as a function from an interval $i$ on $t$ to an interval $j$ on the $q$ dimension (see Table 3). Phases can be distinguished by properties of the domain and/or range. A state is a phase whose range is a point (that is, the smallest interval) on $q$. A process is a phase whose domain and range are extended on $t$ and $q$ respectively. Processes may be monotonic $(\operatorname{Mon}(p))$ or nonmonotonic.

A transition (Trans) is a phase derived from two phases that meet: it is made up of the finish "point" of the first phase and the start "point" of the second phase. This is our solution to the "divided instant" problem described by Mani and Pustejovsky (2012, pg. 60); our solution is similar to that of Hobbs and Pan (2004) (however they distinguish instants from intervals). We divide the "instant" of transition of two phases that meet into the finish point of the first phase and the start point of the second phase. The transition phase is a composite phase made up of those two point phases.

In order to define transitions, we first define start and finish "points" of a temporal interval. We then define start and finish phases of a larger phase, namely the phases whose domains are the start and finish points of the larger phase. A transition phase

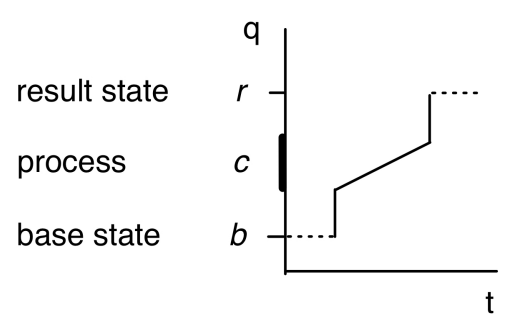

Figure 2: The structure of a telic predicate on the $q$ dimension 
is then defined as a composite phase made up of the finish of the first phase and start of the second phase. A transition phase is not a point interval, but it is the smallest extended interval: that is, there is no interval between the finish point of the first phase and the start point of the second phase, since the two phases meet.

Finally, for convenience we define specific phases in terms of the interval on $q$ that serves as their range; these are $b^{\prime}, c^{\prime}$ and $r^{\prime}$ in Table 3 in the Supplementary Material. Because of the nature of $b, c, r$, it follows that $\operatorname{State}\left(b^{\prime}, i, b, q\right)$, $\operatorname{Process}\left(c^{\prime}, i, c, q\right)$ and $\operatorname{State}\left(r^{\prime}, i, r, q\right)$.

A subevent has an aspectual type. Aspectual types are composite entities composed of one or more phases. The four types of states differ with respect to their domains (time intervals) on $t$, defined on the interval calculus. Unbounded events, that is noninherent states and activities, presuppose that there was a transition from the base state to the asserted phase; the presupposed phase is represented by an existentially quantified predicate. Formalizations of all aspectual types can be found in Table 4 in the Supplementary Material.

\subsection{Events as Force Dynamic Chains of Subevents}

Events expressed by single clauses are informally analyzed as interactions between participants for multiparticipant events. For example, in The rock broke the window, the rock acted on the window. We analyze these force-dynamic relations as relations between subevents that are components of the participant's history. In our example, the rock's contact subevent caused the window's change of state subevent (the specific qualitative state being contributed by the semantics of the verb break). The rock's contact subevent is a component of the rock's history, and likewise the window's change of state event is a component of the window's history.

The unity of an event expressed by a single clause (verb and argument structure construction) is defined by the fact that all subevents of an event are simultaneous, what Croft (2012) calls the temporal unity of events; and by the presence of force dynamic relations between the subevents.

We model the type of incremental change that a participant undergoes, described in section 2, as a property of that participant's subevent, or more precisely the qualitative dimension of that subevent. The types of change described in section 2 are Property change (Prop), Motion (Mot), Mereological change (Mer), Design change (Des), and Internal change (Int). Mereological change falls into four subtypes. Apply represents incremental change of the spatial figure with respect to the ground object, for example paint being gradually applied to a wall. Apply and Remove are inverses, represented by +Mer and -Mer. Cover represents a construal by which the incremental change happens to the spatial ground, for example the wall being gradually covered by the paint. Cover and Uncover are also inverses.

We also provide an analysis of the qualities of subevents of the agent and instrument, not discussed by Croft et al. (2016). Agents interact in physical processes using their body. Most of the time what the agent does is volitional, that is, a process involving mental as well as physical aspects of a person. For now, we model volitionality as the type of action that an agent engages in, that is, the agent's subevent has the property Vol. Instruments interact solely physically, of course, ultimately through some sort of contact. We model the interaction of instruments by attributing the property Contact to the instrument's subevent.

The aspectual annotation of the overall event describes the aspectual type of the theme participant. For this reason, the formalization of the aspectual annotation of the overall event is distinct from the representation of the aspectual type of each subevent. The formalization of the force dynamic annotation that includes the theme participant specifies which participant is the theme. The combination of the aspectual annotation predicate and the force dynamic annotation predicate(s) specifies the aspectual type of the theme participant subevent. The physical force and mental "force" applied by an instrument is dynamic but nonmonotonic. The aspectual type of an agent or instrument subevent varies depending on the aspectual type of the theme: an undirected activity if the overall event is unbounded, an undirected endeavor if the event is bounded and durative, or a semelfactive if the event is punctual.

Formalization of all of the force dynamic types analyzed so far, including external/internal cause, is found in Table 7 in the Supplementary Material. 


\begin{tabular}{|c|c|}
\hline \multicolumn{2}{|c|}{ Aspectual Types/Image Schemas all below $\supset \operatorname{AspTyp}(x, i, j, q)$} \\
\hline Inherent state & $\operatorname{Inhst}(x, i, r, q) \equiv \operatorname{Inherent}(r, q) \& \operatorname{Equal}(i, t)$ \\
\hline Inherent state phase & $\begin{array}{l}\operatorname{InhStPh}(b, i, j, q) \equiv \operatorname{Phase}(b, i, j, q) \&(\exists p, l, m)[\operatorname{Inhst}(p, l, m, q) \& \operatorname{During}(i, l) \& \\
\operatorname{Maps}(p, i, j)]\end{array}$ \\
\hline Incremental accomplishment & $\begin{array}{l}\operatorname{IncrAcc}(x, i, j, q) \equiv \operatorname{Telic}(b, c, r, q) \& x=p_{1}+c^{\prime}+p_{2} \& \operatorname{Mon}\left(c^{\prime}\right) \& \\
\left(\exists b^{\prime}, r^{\prime}\right)\left[\operatorname{Trans}\left(p_{1}, b^{\prime}, c^{\prime}\right) \& \operatorname{Trans}\left(p_{2}, c^{\prime}, r^{\prime}\right)\right]\end{array}$ \\
\hline Undirected endeavor & $\begin{array}{l}\operatorname{UndEnd}(x, i, j, q) \equiv \operatorname{Graded}(b, c, q) \& x=p_{1}+c^{\prime}+p_{2} \& \neg \operatorname{Mon}\left(c^{\prime}\right) \& \\
\left(\exists b^{\prime}\right)\left[\operatorname{Trans}\left(p_{1}, b^{\prime}, c^{\prime}\right) \& \operatorname{Trans}\left(p_{2}, c^{\prime}, b^{\prime}\right)\right]\end{array}$ \\
\hline \multicolumn{2}{|c|}{ Force Dynamic Image Schemas } \\
\hline Volitional & $\begin{array}{l}\text { Volitional }(e, x, y, i) \equiv \text { Component-of }(f, x) \& \text { Component-of }(g, y) \& \\
\text { Subevent }\left(f, i, j, q_{1}\right) \& \text { Subevent }\left(g, i, k, q_{2}\right) \& \text { Force }(f, g) \& \operatorname{Vol}\left(q_{1}\right)\end{array}$ \\
\hline Apply & $\begin{array}{l}\text { Apply }(e, x, y, i) \equiv \text { Theme-of }(e, x) \& \text { Component-of }(g, y) \& \operatorname{Component-of}(h, z) \& \\
\text { Subevent }\left(g, i, j, q_{1}\right) \& \operatorname{InhStPh}\left(h, i, k, q_{2}\right) \& \operatorname{Path}(g, h) \&+\operatorname{Mer}\left(q_{1}\right) \& \operatorname{Exist}\left(q_{2}\right)\end{array}$ \\
\hline \multicolumn{2}{|c|}{ Aspectual Type of Theme Participant } \\
\hline Incremental Accomplishment & $\begin{array}{l}\text { IncrementalAccomplishment }(e, i) \equiv \text { Theme-of }(x, e) \& \text { Component-of }(g, x) \& \\
\operatorname{IncrAcc}(g, i, j, q)\end{array}$ \\
\hline \multicolumn{2}{|c|}{ Predicate Calculus Representation of Example Sentence } \\
\hline $\begin{array}{l}\text { He dumped them into some } \\
\text { baskets. }\end{array}$ & $\begin{array}{l}\text { Dump }(\text { Farmer, Pears, Baskets }) \equiv \operatorname{Component-of}(f, \text { Farmer }) \& \\
\text { Component-of }(g, \text { Pears }) \text { Component-of }(h, \text { Baskets }) \& \operatorname{UndEnd}\left(f, i, j, q_{1}\right) \& \\
\operatorname{IncrAcc}\left(g, i, k, q_{2}\right) \& \operatorname{InhStPh}\left(h, i, l, q_{3}\right) \& \operatorname{Vol}\left(q_{1}\right) \&+\operatorname{Mer}\left(q_{2}\right) \& \operatorname{Exist}\left(q_{3}\right) \& \\
\text { Force }(f, g) \& \operatorname{Path}(g, h)\end{array}$ \\
\hline
\end{tabular}

Table 2: Formalization of aspectual and force dynamic image schemas for example sentence.

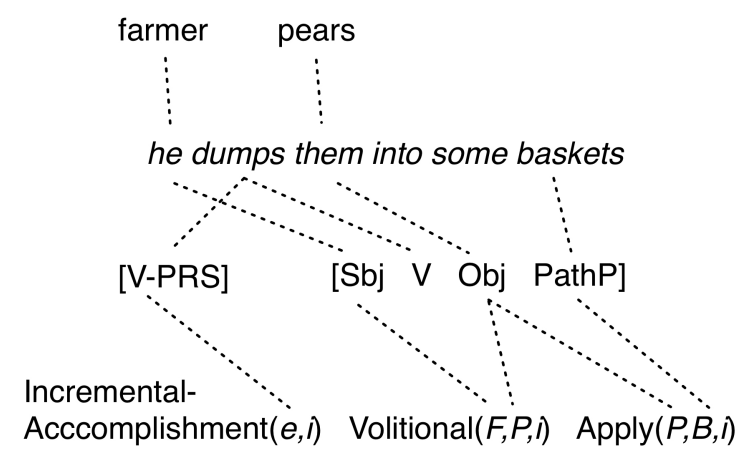

Figure 3: Linking constructions to the semantic representation

\subsection{Deriving the Graphic Representation of the Event Structure of a Sentence}

To illustrate the formalization of the aspectual and force-dynamic decomposition of events, we briefly go through the derivation of the semantic representation of the sentence represented graphically in Figure 1. The first step is extracting the argument structure construction and tense-aspect construction forms from the sentence (not modeled here). The associated meaning is represented by the aspectual and force dynamic annotations, with the arguments of the semantic annotations bound to the participants in the construction; see Figure 3.

The force dynamic annotation, in two parts, can be expanded with the representations in Table 2. A Volitional external cause involves the farmer's volitional subevent in a force relation with the pears' subevent. The pears' subevent involves mereolog- ically moving the pears with respect to the baskets' subevent. The baskets' subevent is simply the phase of the inherent state of existing as an entity with which the pears enter a spatial relationship; this is represented by the inherent state phase formalization in Table 2.

The incremental accomplishment aspectual type is associated with the theme argument, as noted above. The formalization of incremental accomplishments in Table 2 indicates that the $q$ dimension of an incremental accomplishment defines base, center and result intervals. The subevent spans the transition from the base state to the central process and from the central process to the result state, that is, the subevent is bounded; it is also monotonic (see Figure 1).

The agent subevent is specified as an undirected endeavor, since the overall event is temporally bounded. As such, the $q$ dimension defines base and center intervals only. The subevent spans the transition from the base state to the central process and back to the base state.

The predicate calculus representation allows the decompositional event structures and the relations between clausal events to be used for inference using commonsense reasoning axiomatizations such as those in Allen (1984), Hobbs (2005) and Gordon and Hobbs (2017). The predicate calculus representations also specify the structures of the events and their participants to the degree that visualizations can be constructed. These are described in the next section. 


\section{Visualization}

We are also developing a visualization to capture the evolving interactions of participants over time. The basic idea is a modified metro map (Shahaf et al., 2012), in which the lines represent participant histories and the nodes represent interactions among participants, that is, clausal events. Figure 4 presents a visualization of the events, participants and interactions in the passage in Table 1 above.

Clausal events are related to other clausal events through temporal relations and relations of shared participants, as in van Erp et al. (2014). As with other storyline visualizations, temporally sequenced events-Before, After, and Meets in the interval logic — can be arranged horizontally, with sequenced events sharing participants aligned horizontally. Temporally overlapping events-Equal, Overlap, During and Contain-can be arranged vertically. Events whose temporal location is constrained but not totally specified would be situated relative to those events to which they hold temporal relations.

Of course, such metro maps get very tangled very quickly, since coherent narratives normally express many interwoven events with many different combinations of many different participants. Algorithms such as that of Liu et al. (2013) will, we hope, generate visually presentable metro maps of more complex participant interactions over time.

The primary innovation in the visualization is that the interactions between participants in a single clausal event are made explicit, as in Croft (2012) and Croft et al. (2016). That is, the nodes in the metro map visualization are elaborated as interactions between the participants. The roles of participants within a clausal event are kept separate because each participant has its own subevent. The qualitative states and changes of each participant are also explicitly represented. The visualization therefore describes not only the interactions that each participant engages in over the time of the story, but also exactly what they do or what happens to them.

Precise representation of participants and their states in events requires addressing certain issues. A group of participants may act as a unit in some events but separately in other events:

41 [.6] they g [.25] gather all the pears

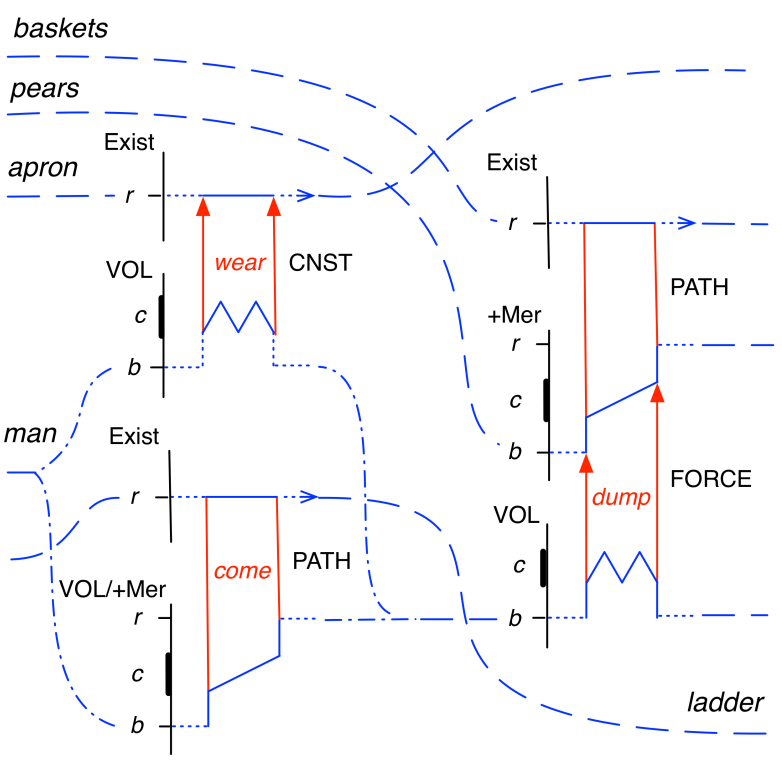

Figure 4: Event Decomposition and Interactions of Participants

42 and put them in the basket,

43 a-nd one of the guys, helps him

44 brush off the dust,

45 [.9] and another guy picks up the rock,

In this case, we must allow the history for the group of three boys to split in order to represent the interaction of individual boys from the group with other entities (the cyclist and the rock).

In other cases, the same participant is playing different roles in two different events at the same time:

140 then he . . takes a pear,

141 after carefully watching the man in the tree.

142 Who's still picking.

The man in the tree is functioning as the target of the watching event in 141 at the same time that he is the agent of the picking event in 142 .

Ideally, there would be a $n$-dimensional representation with all the distinct relevant qualitative dimensions to describe the states of a participant during any given time interval. Of course, this is not easily visualized. In order to represent the distinct qualitative states of the man in the overlapping events, we allow a "virtual split" of the line representing the man's history, representing the 
different qualitative states of being watched and picking. Such virtual splits will be visualized in a distinct way from actual splits as found in passage 41-45 above. In Figure 4, we represent the virtual split of the man by dot-dashed lines.

\section{Conclusion and Future Prospects}

The participant-oriented description of storylines presented here allows for the representation of the qualitative state(s) that each participant is in at any temporal interval in the participant's history, and hence the storyline.

At this point, we have not represented the qualitative states of participants between events. However, some general patterns can easily be implemented. The identification of the theme participant indicates which participant undergoes and change, and the resulting state for that participant, if there is one, can be assumed to hold, other things being equal. Even for the case of events without a result state for the participant, the event can be assumed to persist. The subevents of agent and instrument participants will end at the end of the event; they will return to the base state.

In some cases, grammatical elements provide information that allows one to infer qualitative states between events. For example, in line 142, Who's still picking, the aspectual adverb still indicates that the picking activity described there is a continuation of the same picking activity reported earlier in the narrative. As we model additional tense-aspect constructions, including aspectual adverbs, we will be able to represent the persistence or not of subevents past the reported event.

However, in many cases, the persistence (or lack thereof) of qualitative states can only be inferred using world knowledge. These inferences can be done using the formalization in section 3, combined with representations of relevant commonsense knowledge.

The representation we have developed applies only to events presented as having actually happened in the narrative. Many events in narratives, including news stories, are unrealized in various ways at the point that they are introduced: they may represent planned events, desired events, or events reported with a degree of uncertainty. Even so, they can often be sequenced relative to realized events: planned events follow the current realized event, negated events are "simultaneous" with the actual state of affairs (which is the opposite of the negated event in relevant respects), and so on.

We have not included unrealized events in our annotation and representation. We are developing a model of non-real events using mental spaces (Fauconnier, 1985, 1997) or worlds (McCawley, 1993) in which the non-real events hold in their own mental space/world, and can be related to real events in different ways. Mental spaces would be represented by using the Holds predicate (Allen, 1984; Hobbs, 1985b), with a world argument $w$ added, relativized to an agent holding the belief or intending a plan etc.: $\operatorname{Holds}(e, a, w)$. The relationship between mental spaces and their basis in reality will be modeled following Clark (1996). Other predicates represent relations between mental spaces or between agents and their mental spaces, building on Fauconnier $(1985,1997)$ and McCawley (1993).

\section{Acknowledgments}

The research was supported in part by grant HDTRA1-15-1-0063 by the Defense Threat Reduction Agency to the first author.

\section{References}

James Allen. 1984. Towards a general theory of action and time. Artificial Intelligence 23:123-154.

Tommaso Caselli and Piek Vossen. 2012. The Storyline Annotation and Representation Scheme (StaR): a proposal. In Proceedings of the 2nd Workshop on Computing News Storylines, Stroudsburg, Penn: Association for Computational Linguistics, pages 6772 .

Wallace Chafe, editor. 1980. The pear stories. New York: Ablex.

Herbert H. Clark. 1996. Using language. Cambridge: Cambridge University Press.

William Croft. 2012. Verbs: aspect and causal structure. Oxford University Press.

William Croft, Pavlína Pešková, and Michael Regan. 2016. Annotation of causal and aspectual structure of events in RED: a preliminary report. In 4th Events Workshop, 15th Annual Conference of the North American Chapter of the Association of Computational Linguistics: Human Language Technologies (NAAACL-HLT 2016), Stroudsburg, Penn: Association for Computational Linguistics, pages 817.

David Dowty. 1991. Thematic proto-roles and argument selection. Language 67:547-619. 
Gilles Fauconnier. 1985. Mental Spaces. Cambridge, Mass: MIT Press.

Gilles Fauconnier. 1997. Mappings in thought and language. Cambridge: Cambridge University Press.

James Forsyth. 1970. A grammar of aspect: usage and meaning in the Russian verb. Cambridge: Cambridge University Press.

Adele E. Goldberg. 1995. Constructions: A Construction Grammar Approach to Argument Structure. Chicago: University of Chicago Pres.

Adele E. Goldberg. 2006. Constructions at Work: The Nature of Generalization in Language. Oxford: Oxford University Press.

Andrew S. Gordon and Jerry R. Hobbs. 2017. A Formal Theory of Commonsense Psychology: How People Think People Think. Cambridge University Press, to appear.

Jennifer Hay, Christopher Kennedy, and Beth Levin. 1999. Scalar structure underlies telicity in "degree achievements". In Tanya Matthews and Devon Strolovitch, editors, Proceedings of SALT, Ithaca: Cornell University Press, volume 9, pages 127-144.

Jerry R. Hobbs. 1985a. Granularity. In Proceedings of the Ninth International Joint Conference on Artificial Intelligence, Volume 1, San Francisco, Calif.: Morgan Kaugmann, pages 432-435.

Jerry R. Hobbs. 1985b. Ontological promiscuity. In Proceedings of the 12th Annual Meeting of the Association for Computational Linguistics, Stroudsburg, Penn: Association for Computational Linguistics.

Jerry R. Hobbs. 2005. Toward a useful concept of causality for lexical semantics. Journal of Semantics 22:181-209.

Jerry R. Hobbs and Feng Pan. 2004. An ontology of time for the semantic web. ACM Transactions on Asian Language Information Processing 3(1):6685.

Syeohun Im and James Pustejovsky. 2010. Annotating lexically entailed subevents for textual inference tasks. In Proceedings of the 23rd International Florida Artificial Intelligence Research Society Conference (FLAIRS-23), Daytona Beach, Florida, pages 204-209.

Beth Levin and Malka Rappaport Hovav. 2005. Argument realization. Cambridge: Cambridge University Press.

Shixia Liu, Yingcai Wu, Enxun Wei, Mengchen Liu, and Yang Liu. 2013. Storyflow: tracking the evolution of stories. IEEE Transactions on Visualization and Computer Graphics 19(12):2436-2445.

Inderjeet Mani and James Pustejovsky. 2012. Interpreting motion: grounded representations for spatial language. Oxford: Oxford University Press.
James D. McCawley. 1993. Everything that linguists have always wanted to know about logic (but were ashamed to ask). Chicago: University of Chicago Press, 2 edition.

Srini Narayanan. 1997. Knowledge-based action representations for metaphor and aspect (KARMA). $\mathrm{Ph}$.D. thesis, Department of Computer Science, University of California at Berkeley.

Roxane Segers, Piet Vossen, Marco Rospocher, Luciano Serafini, Egoitz Laparra, and German Rigau. 2015. ESO: a frame-based ontology for events and implied situations. In Proceedings of the MAPLEX2015 Workshop.

Dafna Shahaf, Carlos Guestrin, and Eric Horvitz. 2012. Trains of thought: generating information maps. In Proceedings of the 21st International Conference on World Wide Web, New York: Association of Computing Machinery, pages 899-908.

Leonard Talmy. 1983. How language structures space. In Herbert L. Pick (Jr.) and Linda P. Acredolo, editors, Spatial orientation: theory, research and application, New York: Plenum Press, pages 225-282.

Leonard Talmy. 1988. Force dynamics in language and cognition. Cognitive Science 2:49-100.

Marieke van Erp, Gleb Satyukov, Piek Vossen, and Marit Nijssen. 2014. Discovering and visualizing stories in the news. In Proceedings of the 9th Language Resources and Evaluation Conference (LREC2014), European Language Resources Association, pages 3277-3282.

Zeno Vendler. 1957. Verbs and times. The Philosophical Review 66:143-60. 


\section{A Supplementary Material: Annotation and Formalization of Aspect and Force Dynamic Structure of Events}

\begin{tabular}{|c|c|}
\hline Structure of q: & Phases: \\
\hline Inherent $(r, q) \equiv \operatorname{Pnt}(r) \& \operatorname{Equal}(r, q)$ & Phase $(p, i, j, q) \equiv$ Function $(p, i, j) \&$ Interval-on $(i, t) \&$ Interval-on $(j, q)$ \\
\hline $\begin{array}{l}\text { Complementary }(b, r, q) \equiv q=b+r \& \operatorname{Pnt}(b) \& \\
\operatorname{Pnt}(r) \& \operatorname{Meets}(b, r)\end{array}$ & $\begin{array}{l}b^{\prime}: \quad \text { Phase }\left(b^{\prime}, i, b, q\right) \&(\text { Complementary }(b, r, q) \vee \operatorname{Graded}(b, c, q) \vee \\
\text { Telic }(b, c, r, q))\end{array}$ \\
\hline $\begin{array}{l}\operatorname{Graded}(b, c, q) \equiv q=b+c \& \operatorname{Pnt}(b) \& \operatorname{Ext}(c) \& \\
\operatorname{Meets}(b, c)\end{array}$ & $c^{\prime}:$ Phase $\left(c^{\prime}, i, c, q\right) \&(\operatorname{Graded}(b, c, q) \vee \operatorname{Telic}(b, c, r, q))$ \\
\hline $\begin{array}{l}\text { Telic }(b, c, r, q) \equiv q=b+c+r \& \operatorname{Pnt}(b) \& \operatorname{Ext}(c) \\
\& \operatorname{Pnt}(r) \& \operatorname{Meets}(b, c) \& \operatorname{Meets}(b, r)\end{array}$ & 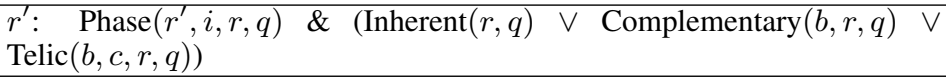 \\
\hline \multicolumn{2}{|l|}{ Transitions: } \\
\hline Start point: $\operatorname{Spt}(x, i) \equiv \operatorname{Starts}(x, i) \& \operatorname{Pnt}(x)$ & Finish point: $\operatorname{Fpt}(x, i) \equiv \operatorname{Finishes}(x, i) \& \operatorname{Pnt}(x)$ \\
\hline $\begin{array}{l}\text { Start phase: } \operatorname{Sph}(s, p) \equiv \operatorname{Phase}(s, i, j, q) \& \\
\operatorname{Phase}(p, k, l, q) \& \operatorname{Spt}(i, k) \& \operatorname{Maps}(p, i, j)\end{array}$ & $\begin{array}{l}\text { Finish phase: } \operatorname{Fph}(f, p) \equiv \operatorname{Phase}(f, i, j, q) \& \operatorname{Phase}(p, k, l, q) \& \operatorname{Fpt}(i, k) \\
\& \operatorname{Maps}(p, i, j)\end{array}$ \\
\hline $\begin{array}{l}\text { Transition phase: } \operatorname{Trans}\left(p, p_{1}, p_{2}\right) \equiv \operatorname{Phase}(p \\
\operatorname{Fph}\left(f, p_{1}\right) \& \operatorname{Sph}\left(s, p_{2}\right) \& p=f+s\end{array}$ & $j, q) \&$ Phase $\left(p_{1}, k, l, q\right) \& \operatorname{Phase}\left(p_{2}, m, n, q\right) \& \operatorname{Meets}(k, m) \&$ \\
\hline
\end{tabular}

Table 3: Structure of $q$ dimensions and types of phases. These axioms and definitions underlie the phasal geometrical model of aspect.

\begin{tabular}{|c|c|}
\hline \multicolumn{2}{|c|}{ Aspectual types/image schemas } \\
\hline \multicolumn{2}{|l|}{ Inherent States } \\
\hline Full state & $\operatorname{Inhst}(x, i, r, q) \equiv \operatorname{Inherent}(r, q) \& \operatorname{Equal}(i, t)$ \\
\hline Phase of state & $\begin{array}{l}\operatorname{InhStPhase}(b, i, k, q) \equiv \operatorname{Phase}(b, i, k, q) \&(\exists p, l, m)(\operatorname{Inhst}(p, l, m, q) \& \operatorname{During}(i, l) \& \\
\operatorname{Maps}(p, i, k))\end{array}$ \\
\hline \multicolumn{2}{|c|}{ Noninherent States } \\
\hline Reversible & $\operatorname{RevSt}(x, i, r, q) \equiv$ Complementary $(b, r, q) \& \operatorname{Ext}(i) \&\left(\exists p, b^{\prime}\right) \operatorname{Trans}\left(p, b^{\prime}, x\right)$ \\
\hline Irreversible & $\operatorname{IrrSt}(x, i, r, q) \equiv$ Complementary $(b, r, q) \&$ Finishes $(i, t) \&\left(\exists p, b^{\prime}\right) \operatorname{Trans}\left(p, b^{\prime}, x\right)$ \\
\hline Point & $\operatorname{PntSt}(x, i, r, q) \equiv$ Complementary $(b, r, q) \& \operatorname{Pnt}(i) \&\left(\exists p, b^{\prime}\right) \operatorname{Trans}\left(p, b^{\prime}, x\right)$ \\
\hline \multicolumn{2}{|r|}{ 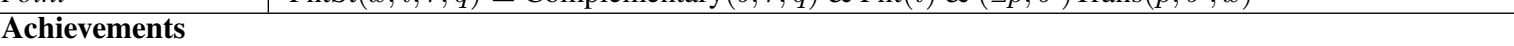 } \\
\hline Directed & $\operatorname{DirAch}(x, i, j, q) \equiv$ Complementary $(b, r, q) \&\left(\exists b^{\prime}, r^{\prime}\right) \operatorname{Trans}\left(x, b^{\prime}, r^{\prime}\right)$ \\
\hline Cyclic & $\begin{array}{l}\operatorname{CycAch}(x, i, j, q) \equiv \text { Complementary }(b, r, q) \& x=p_{1}+p_{2} \&\left(\exists b^{\prime}, r^{\prime}\right)\left(\operatorname{Trans}\left(p_{1}, b^{\prime}, r^{\prime}\right) \&\right. \\
\left.\operatorname{Trans}\left(p_{2}, r^{\prime}, b^{\prime}\right)\right) \& \text { OverlapPnt }\left(p_{1}, p_{2}\right)\end{array}$ \\
\hline \multicolumn{2}{|l|}{ Activities } \\
\hline Undirected & $\operatorname{UndAct}(x, i, c, q) \equiv \operatorname{Graded}(b, c, q) \& \neg \operatorname{Mon}(x) \&\left(\exists p, b^{\prime}\right) \operatorname{Trans}\left(p, b^{\prime}, x\right)$ \\
\hline Directed & $\operatorname{DirAct}(x, i, c, q) \equiv \operatorname{Graded}(b, c, q) \& \operatorname{Mon}(x) \&\left(\exists p, b^{\prime}\right) \operatorname{Trans}\left(p, b^{\prime}, x\right)$ \\
\hline \multicolumn{2}{|c|}{ Accomplishments } \\
\hline Incremental & $\begin{array}{l}\operatorname{IncrAcc}(x, i, j, q) \equiv \operatorname{Telic}(b, c, r, q) \& x=p_{1}+c^{\prime}+p_{2} \& \operatorname{Mon}\left(c^{\prime}\right) \&\left(\exists b^{\prime}, r^{\prime}\right)\left(\operatorname{Trans}\left(p_{1}, b^{\prime}, c^{\prime}\right)\right. \\
\left.\& \operatorname{Trans}\left(p_{2}, c^{\prime}, r^{\prime}\right)\right)\end{array}$ \\
\hline Nonincremental & $\begin{array}{l}\text { NonincrAcc }(x, i, j, q) \equiv \operatorname{Telic}(b, c, r, q) \quad \& \quad x \quad=\quad p_{1}+c^{\prime}+p_{2} \quad \& \quad \neg \operatorname{Mon}\left(c^{\prime}\right) \& \\
\left(\exists b^{\prime}, r^{\prime}\right)\left(\operatorname{Trans}\left(p_{1}, b^{\prime}, c^{\prime}\right) \& \operatorname{Trans}\left(p_{2}, n, c^{\prime}, r^{\prime}\right)\right)\end{array}$ \\
\hline \multicolumn{2}{|r|}{ (2) } \\
\hline Undirected & $\begin{array}{l}\operatorname{UndEnd}(x, i, j, q) \equiv \operatorname{Graded}(b, c, q) \& x=p_{1}+c^{\prime}+p_{2} \& \neg \operatorname{Mon}\left(c^{\prime}\right) \&\left(\exists b^{\prime}\right)\left(\operatorname{Trans}\left(p_{1}, b^{\prime}, c^{\prime}\right)\right. \\
\left.\& \operatorname{Trans}\left(p_{2}, c^{\prime}, b^{\prime}\right)\right)\end{array}$ \\
\hline Directed & $\begin{array}{l}\operatorname{DirEnd}(x, i, j, q) \equiv \operatorname{Graded}(b, c, q) \& x=p_{1}+c^{\prime}+p_{2} \& \operatorname{Mon}\left(c^{\prime}\right) \&\left(\exists b^{\prime}\right)\left(\operatorname{Trans}\left(p_{1}, b^{\prime}, c^{\prime}\right) \&\right. \\
\left.\operatorname{Trans}\left(p_{2}, c^{\prime}, b^{\prime}\right)\right)\end{array}$ \\
\hline
\end{tabular}

Table 4: Definitions of aspectual contours as composites of phases. The terms in the left hand column make up the annotation of the aspectual type of the overall event. The aspectual type of the overall event is identical to the aspectual type of the subevent of the theme participant; see Table 7. This mapping is done by rules of the type illustrated in the formalization of the example sentence in Table 2. 


\begin{tabular}{|l|l|l|}
\hline \multirow{2}{*}{} & Direct & Inverse \\
\cline { 2 - 3 } & Force (contact, force exertion) & Resist (maintain) \\
\hline \hline Theme & Direct & Reverse \\
\hline Property & Change of State & \\
\hline Path & Motion (directed motion, manner of motion) & \\
\hline \multirow{2}{*}{ Mereological } & Apply (application, combining) & Remove (removal, separation) \\
\cline { 2 - 3 } & Cover ( covering, filling) & Uncover (uncovering, emptying) \\
\hline \multirow{2}{*}{ Design } & Create & \\
\cline { 2 - 3 } & Form & \\
\hline \multirow{2}{*}{ Existence } & Internal & Dynamic Texture \\
\cline { 2 - 3 } & Location & \\
\hline
\end{tabular}

Table 5: Force-dynamic image schemas for annotation: theme change type. The terms in the second and third columns make up the annotation.

\begin{tabular}{|l|l|l|}
\hline External Cause & Example \\
\hline \hline Autonomous & no external cause & Paint spilled onto the floor. \\
\hline Self-Volitional & $\begin{array}{l}\text { no external cause; theme argument brings about } \\
\text { action volitionality }\end{array}$ & Wanda ran out of the room. \\
\hline Physical & external physical cause & The baseball shattered the window. \\
\hline Volitional & external volitional cause; no distinct instrument & I painted the wall. \\
\hline Instrumental & external volitional cause with distinct instrument & I painted the wall with a roller. \\
\hline
\end{tabular}

Table 6: External/Internal cause. The terms in the first column make up the annotation.

\begin{tabular}{|c|c|}
\hline \multicolumn{2}{|c|}{ Initial part of causal chain } \\
\hline Volitional & $\begin{array}{l}\text { Volitional }(x, y, i) \equiv \text { Component-of }(f, x) \& \text { Component-of }(g, y) \& \text { Subevent }\left(f, i, j, q_{1}\right) \& \\
\text { Subevent }\left(g, i, k, q_{2}\right) \& \operatorname{Force}(f, g) \& \operatorname{Vol}\left(q_{1}\right)\end{array}$ \\
\hline Physical & $\begin{array}{l}\text { Physical }(x, y, i) \equiv \text { Component-of }(f, x) \& \text { Component-of }(g, y) \& \text { Subevent }\left(f, i, j, q_{1}\right) \text { \& } \\
\text { Subevent }\left(g, i, k, q_{2}\right) \& \text { Force }(f, g) \& \operatorname{Cont}\left(q_{1}\right)\end{array}$ \\
\hline Instrument & $\begin{array}{l}\text { Instrument }(x, y, z, i) \equiv \text { Component-of }(f, x) \& \text { Component-of }(g, y) \text { \& Component-of }(h, z) \& \\
\text { Subevent }\left(f, i, j, q_{1}\right) \& \operatorname{Subevent}\left(g, i, k, q_{2}\right) \& \operatorname{Subevent}\left(h, i, l, q_{3}\right) \& \operatorname{Force}(f, h) \& \operatorname{Vol}\left(q_{1}\right) \& \\
\text { Force }(h, g) \& \operatorname{Cont}\left(q_{3}\right)\end{array}$ \\
\hline Self-volitional & Self-Volitional $(x, i) \equiv$ Component-of $(f, x) \&$ Subevent $(f, i, j, q) \& \operatorname{Vol}(q)$ \\
\hline \multicolumn{2}{|c|}{ Central part of causal chain } \\
\hline COS & $\operatorname{COS}(x, i) \equiv$ Theme-of $(e, x) \&$ Component-of $(f, x) \&$ Subevent $(f, i, j, q) \& \operatorname{Prop}(q)$ \\
\hline Motion & $\begin{array}{l}\text { Motion }(x, y, i) \quad \equiv \quad \text { Theme-of }(e, x) \quad \& \quad \text { Component-of }(f, x) \quad \& \quad \text { Component-of }(g, y) \quad \& \\
\text { Subevent }\left(f, i, j, q_{1}\right) \& \operatorname{InhStPh}\left(g, i, k, q_{2}\right) \& \operatorname{Path}(f, g) \& \operatorname{Mot}\left(q_{1}\right)\end{array}$ \\
\hline Apply & $\begin{array}{l}\operatorname{Apply}(x, y, i) \equiv \text { Theme-of }(e, x) \quad \& \quad \text { Component-of }(f, x) \quad \& \quad \text { Component-of }(g, y) \quad \& \\
\text { Subevent }\left(f, i, j, q_{1}\right) \& \operatorname{InhStPh}\left(g, i, k, q_{2}\right) \& \operatorname{Path}(f, g) \&+\operatorname{Mer}\left(q_{1}\right) \& \operatorname{Exist}\left(q_{2}\right)\end{array}$ \\
\hline Remove & $\begin{array}{l}\text { Remove }(x, y, i) \equiv \text { Theme-of }(e, x) \quad \& \quad \text { Component-of }(f, x) \quad \& \quad \text { Component-of }(g, y) \quad \& \\
\text { Subevent }\left(f, i, j, q_{1}\right) \& \operatorname{InhStPh}\left(g, i, k, q_{2}\right) \& \operatorname{Path}(f, g) \&-\operatorname{Mer}\left(q_{1}\right) \& \operatorname{Exist}\left(q_{2}\right)\end{array}$ \\
\hline Cover & $\begin{array}{l}\text { Cover }(x, y, i) \equiv \text { Theme-of }(e, y) \quad \& \quad \text { Component-of }(f, x) \quad \& \quad \text { Component-of }(g, y) \\
\text { Subevent }\left(f, i, j, q_{1}\right) \& \operatorname{Subevent}\left(g, i, k, q_{2}\right) \& \operatorname{Path}(f, g) \&+\operatorname{Mer}\left(q_{2}\right) \& \operatorname{Int}\left(q_{1}\right)\end{array}$ \\
\hline Uncover & $\begin{array}{l}\text { Uncover }(x, y, i) \equiv \text { Theme-of }(e, y) \quad \& \text { Component-of }(f, x) \quad \& \text { Component-of }(g, y) \\
\text { Subevent }\left(f, i, j, q_{1}\right) \& \text { Subevent }\left(g, i, k, q_{2}\right) \& \operatorname{Path}(f, g) \&-\operatorname{Mer}\left(q_{2}\right) \& \operatorname{Int}\left(q_{1}\right)\end{array}$ \\
\hline Create & Create $(x, i) \equiv$ Theme-of $(e, x) \&$ Component-of $(f, x) \&$ Subevent $(g, i, j, q) \& \operatorname{Des}(q)$ \\
\hline Form & $\begin{array}{l}\text { Form }(x, y, i) \equiv \quad \text { Theme-of }(e, y) \quad \& \quad \text { Component-of }(f, x) \quad \& \quad \text { Component-of }(g, y) \\
\text { Subevent }\left(f, i, j, q_{1}\right) \& \text { Subevent }\left(g, i, k, q_{2}\right) \& \text { Transform }(f, g) \& \operatorname{Des}\left(q_{2}\right) \& \operatorname{Int}\left(q_{1}\right)\end{array}$ \\
\hline Internal & Internal $(x, i) \equiv$ Theme-of $(e, x) \&$ Component-of $(f, x) \&$ Subevent $(f, i, j, q) \& \operatorname{Int}(q)$ \\
\hline Location & $\begin{array}{l}\text { Location }(x, y, i) \equiv \text { Theme-of }(e, x) \quad \& \text { Component-of }(f, x) \quad \& \text { Component-of }(g, y) \\
\text { Subevent }\left(f, i, j, q_{1}\right) \& \operatorname{InhStPh}\left(g, i, k, q_{2}\right) \& \operatorname{Path}(f, g) \& \operatorname{Int}\left(q_{1}\right) \& \operatorname{Exist}\left(q_{2}\right)\end{array}$ \\
\hline Dynamic Texture & $\begin{array}{l}\text { DynamicTexture }(x, y, i) \equiv \text { Theme-of }(e, y) \& \text { Component-of }(f, x) \& \text { Component-of }(g, y) \& \\
\text { Subevent }\left(f, i, j, q_{1}\right) \& \text { Subevent }\left(g, i, k, q_{2}\right) \& \operatorname{Path}(f, g) \& \operatorname{Int}\left(q_{2}\right) \& \operatorname{Int}\left(q_{1}\right)\end{array}$ \\
\hline
\end{tabular}

Table 7: Formal definitions of event types. The terms in the first column correspond to the force dynamic annotations in Tables 5 and 6 . The aspectual type of the Theme-of argument is the aspectual type of the entire event. The the aspectual types of subevents are determined by the overall aspectual type of the event, based on rules not included here for reasons of space. 